\title{
CMB polarization systematics due to beam asymmetry: Impact on inflationary science
}

\author{
Meir Shimon, ${ }^{1}$ Brian Keating, ${ }^{1}$ Nicolas Ponthieu, ${ }^{2}$ and Eric Hivon ${ }^{3,4}$ \\ ${ }^{1}$ Center for Astrophysics and Space Sciences, University of California, San Diego, \\ 9500 Gilman Drive, La Jolla, California, 92093-0424, USA \\ ${ }^{2}$ Institut d'Astrophysique Spatiale, Bat. 121, Universite Paris XI, 91405 Orsay Cedex, France \\ ${ }^{3}$ California Institute of Technology, Pasadena, California 91125, USA \\ ${ }^{4}$ Institut d'Astrophysique de Paris, 98 bis boulevard Arago, F-75014 Paris, France \\ (Received 10 September 2007; revised manuscript received 18 March 2008; published 7 April 2008)
}

\begin{abstract}
Cosmic microwave background (CMB) polarization provides a unique window into cosmological inflation; the amplitude of the $B$-mode polarization from last scattering is uniquely sensitive to the energetics of inflation. However, numerous systematic effects arising from optical imperfections can contaminate the observed $B$-mode power spectrum. In particular, systematic effects due to the coupling of the underlying temperature and polarization fields with elliptical or otherwise asymmetric beams yield spurious systematic signals. This paper presents a nonperturbative analytic calculation of some of these signals. We show that results previously derived in real space can be generalized, formally, by including infinitely many higher-order corrections to the leading order effects. These corrections can be summed and represented as analytic functions when a fully Fourier-space approach is adopted from the outset. The formalism and results presented in this paper were created to determine the susceptibility of CMB polarization probes of the primary gravitational wave signal but can be easily extended to the analysis of gravitational lensing of the CMB.
\end{abstract}

DOI: 10.1103/PhysRevD.77.083003

PACS numbers: $98.70 . V_{c}$

\section{INTRODUCTION}

Upcoming cosmic microwave background (CMB) polarization experiments are poised to immensely improve our understanding of the early Universe. The significance of polarization lies not only in the fact that it increases the amount of data provided by temperature anisotropy alone but also because it is more sensitive to several physical processes which took place in the early Universe, e.g., the gravitational wave background produced during inflation (Seljak and Zaldarriaga [1] and Kamionkowski, Kosowsky, and Stebbins [2]); and reionization (Zaldarriaga [3] and Fan, Carilli, and Keating [4]). Partially (but not only) due to this fact, $\mathrm{CMB}$ polarization has a unique dependence on the basic cosmological parameters. This feature can be used to remove some of the degeneracies afflicting cosmological parameter estimation from temperature anisotropy alone. One of the CMB polarization's main features is the dependence of its $B$-mode (curl-like polarization) on the amplitude of the stochastic gravitational wave background generically predicted by inflationary models. Because of its faintness, the $B$-mode polarization is prone to degradation by various systematic effects on a wide range of scales and it is important to remove these spurious contributions. This must be done in addition to controlling the various systematics induced by diffuse galactic emission (Amblard, Cooray, and Kaplinghat [5]) and contamination from $E-B$ mixing due to partial sky coverage (Lewis, Challinor, and Turok [6], de Oliveira-Costa and Tegmark [7], and Brown, Castro, and Taylor [8]).

This paper describes an analytic approach to assess systematics induced by imperfections of the polarimeter's main beam. Several effects which contaminate the power spectra stem from temperature and polarization variations over scales comparable to the beamwidth (Hu, Hedman, and Zaldarriaga [9], Ponthieu [10], Rosset et al. [11], and O'Dea, Challinor, and Johnson [12]); these effects can be modeled and characterized by the spurious $C_{l}$ they produce.

Our results are power spectra presented as a combination of the underlying power spectra with mixing coefficients which are infinite sums of analytic functions. In practice however, this infinite series must be truncated and therefore our result is effectively equivalent to a series expansion. As expected, for small beam imperfections we find that the higher-order corrections contribute negligibly to the spurious polarization. We define the small parameters characterizing the systematic effects in Table I. They are;

TABLE I. Definitions of the parameters associated with the systematic effects. Subscripts 1 and 2 refer to the first and second beams, respectively. The differential gain parameter $g$ and the beam rotation parameter $\varepsilon$ are not related to the beam shape and therefore are not defined in Eqs. (7) and (8), but rather are global parameters which define beam mismatch. We defer the exact definitions of these parameters to the relevant sections.

\begin{tabular}{lcc}
\hline Effect & Parameter & Definition \\
\hline Gain & $g$ & $g_{1}-g_{2}$ \\
\hline Monopole & $\mu$ & $\frac{\sigma_{1}-\sigma_{2}}{\sigma_{1}+\sigma_{2}}$ \\
\hline Dipole & $\rho$ & $\rho_{1}-\rho_{2}$ \\
\hline Quadrupole & $e$ & $\frac{\sigma_{x}-\sigma_{y}}{\sigma_{x}+\sigma_{y}}$ \\
\hline Rotation & $\varepsilon$ & $\frac{1}{2}\left(\varepsilon_{1}+\varepsilon_{2}\right)$ \\
\hline \hline
\end{tabular}


the gain factor $g$, the differential beamwidth of the beams $\mu$, the differential pointing $\rho$, the beam ellipticity $e$, and the beam rotation $\varepsilon$.

This paper is organized as follows; in Sec. II we present the basic mathematical formalism of spin-weighted fields used to characterize the systematic effects, beam convolution, and our analytic results for the temperaturepolarization leakage and polarization conversion in a single beam. Ultimately, we consider bolometric polarimetry which is most conveniently described using the Stokes parameters (e.g, Masi et al. [13]) $I, Q$, and $U(V=0$ for $\mathrm{CMB}$ polarization) as opposed to the Jones matrix formalism which is particularly useful for describing coherent polarimeters (Hu, Hedman, and Zaldarriaga [9] and O'Dea, Challinor, and Johnson [12]). These parameters are derived from differences in intensity in the Gaussian 2D polarized beam response function for each polarization. Our twobeam experiment and the induced power spectra are derived in Sec. III and the numerical results for the $B$-mode power spectrum are described in Sec. IV. We end with a discussion of the impact of these effects in Sec. V.

\section{MATHEMATICAL FORMALISM}

We work entirely in Fourier space and begin with the expansion of both temperature and $Q$ and $U$ Stokes parameters in plane waves. Since all the effects considered here are due to the beam asymmetry, and the beamwidths are typically on the degree or subdegree scale, we can safely employ the flat-sky approximation as far as effects related to the beam shape and size are considered. The underlying physical power spectrum is calculated by CAMB (Lewis, Challinor, and Lasenby [14]) using the full sky. The temperature and other (integer-spin) combinations of the Stokes parameters are expanded in harmonic space as follows (e.g. Zaldarriaga and Seljak [15]):

$$
T(\hat{n})=\sum_{l, m} a_{l m} Y_{l m}(\hat{n}) \quad(Q \pm i U)(\hat{n})=\sum_{l, m} a_{ \pm 2, l m \pm 2} Y_{l m}(\hat{n}),
$$

where the expansion coefficients of the spin \pm 2 polarization parameters can be presented in terms of the $E$ and $B$ polarization modes

$$
a_{ \pm 2, l m}=E_{l m} \pm i B_{l m}
$$

$E$ and $B$ are scalar and pseudoscalar under parity (having even and odd parities), respectively, and are sometimes referred to as the "electric" and "magnetic" (or "gradient" and "curl") polarization components. In the flatsky approximation, Eq. (1) becomes

$$
\begin{aligned}
T(\mathbf{x}) & =\frac{1}{2 \pi} \int T_{l} e^{i l \cdot \mathbf{x}} d^{2} \boldsymbol{l} \\
X^{ \pm}(\mathbf{x}) & \equiv(Q \pm i U)(\mathbf{x}) \\
& =\frac{1}{2 \pi} \int\left(E_{l} \pm i B_{l}\right) e^{i l \cdot \mathbf{x}} e^{ \pm 2 i\left(\phi_{l}-\phi_{\mathbf{x}}\right)} d^{2} \boldsymbol{l},
\end{aligned}
$$

where $T_{l}, E_{l}$, and $B_{l}$ are the Fourier components which are functions of the wave vector $l$ only.

Since in real space the temperature and polarization patterns are convolved with the beams, these expressions are simply the product of their Fourier transforms in Fourier space. We restrict the discussion to an elliptical Gaussian beam (with major and minor axes $\sigma_{x}$ and $\sigma_{y}$ )

$$
B(\mathbf{x})=\frac{1}{2 \pi \sigma_{x} \sigma_{y}} \exp \left(-\frac{\left(x-\rho_{x}\right)^{2}}{2 \sigma_{x}^{2}}-\frac{\left(y-\rho_{y}\right)^{2}}{2 \sigma_{y}^{2}}\right)
$$

and its Fourier transform is

$$
\tilde{B}(\boldsymbol{l})=\exp \left(-\frac{l_{x}^{2} \sigma_{x}^{2}}{2}-\frac{l_{y}^{2} \sigma_{y}^{2}}{2}+i \boldsymbol{l} \cdot \rho\right) .
$$

The pointing error merely shifts the phase of the beam representation in Fourier space. It is useful to switch to polar coordinates at this point

$$
\begin{gathered}
l_{x}=l \cos \left(\phi_{l}+\psi-\alpha\right) \quad l_{y}=l \sin \left(\phi_{l}+\psi-\alpha\right) \\
\rho_{x}=\rho \cos \theta \quad \rho_{y}=\rho \sin \theta,
\end{gathered}
$$

where the angles $\psi, \alpha$, and $\theta$ are defined below. The Fourier representation of the beam [Eq. (5)] then becomes

$$
\tilde{B}(\boldsymbol{l}) d^{2} \mathbf{l}=e^{-y-z \cos 2\left(\phi_{l}+\psi-\alpha\right)+i l \rho \cos \left(\phi_{l}-\alpha-\theta+\psi\right)} l d l d \phi_{l},
$$

where

$$
y \equiv \frac{l^{2}}{4}\left(\sigma_{x}^{2}+\sigma_{y}^{2}\right) \quad z \equiv \frac{l^{2}}{4}\left(\sigma_{x}^{2}-\sigma_{y}^{2}\right) .
$$

The definitions of the parameters in terms of the mean beamwidth $\sigma$, differential beamwidth $\mu$, and ellipticity $e$, are given in Table II. Employing the expansion of 2D plane waves in terms of cylindrical Bessel functions

$$
e^{i l \rho \cos \left(\phi_{l}-\phi_{\rho}\right)}=\sum_{n=-\infty}^{n=\infty} i^{n} J_{n}(l \rho) e^{i n\left(\phi_{l}-\phi_{\rho}\right)}
$$

the definition of modified Bessel function

$$
I_{n}(z)=i^{-n} J_{n}(i z)
$$

and the symmetry relation

$$
J_{-n}(z)=(-1)^{n} J_{n}(z),
$$

Eq. (7) becomes 


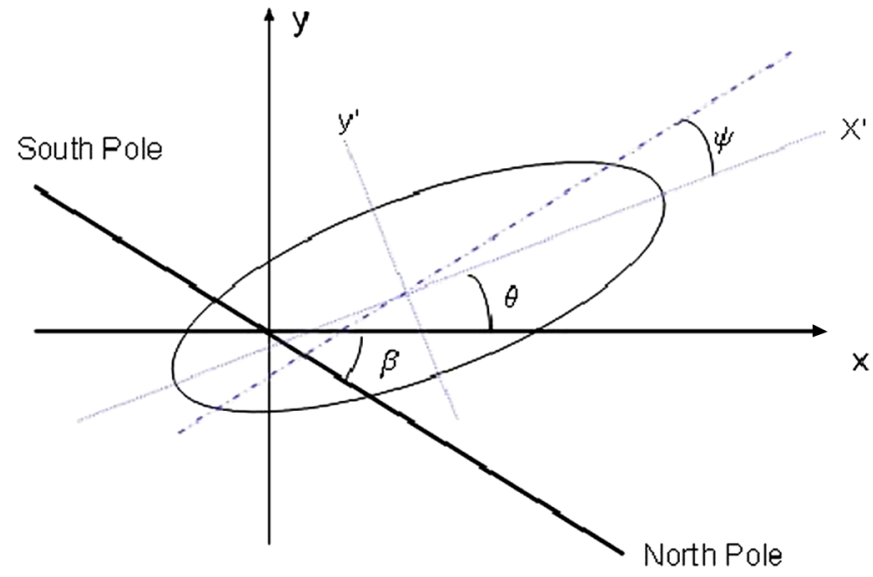

FIG. 1 (color online). The beam profile with the angles $\beta, \theta$, and $\psi$ shown for a single beam. The horizontal $\mathrm{x}$ and vertical $\mathrm{y}$ axes are fixed to the focal plane. The axis of polarization sensitivity makes an angle $\psi$ with the ellipse major axis.

$$
\begin{aligned}
\tilde{B}(\boldsymbol{l})= & e^{-y} \sum_{n=-\infty}^{\infty} \sum_{m=-\infty}^{\infty} i^{2 m+n} I_{m}(z) J_{n}(l \rho) \\
& \times e^{i(2 m+n) \psi-i n \theta} e^{i(2 m+n)\left(\phi_{l}-\alpha\right)} \\
\equiv & \sum_{n=-\infty}^{\infty} \sum_{m=-\infty}^{\infty} B_{m, n} e^{i(2 m+n)\left(\phi_{l}-\alpha\right)},
\end{aligned}
$$

where $\alpha \equiv \beta+\theta+\psi$ is the angle of the polarization axis in some coordinate system fixed to the sky (Fig. 1). We will employ this relation repeatedly in this work.

\section{DUAL POLARIZED BEAM EXPERIMENT}

We represent the data collected by the detectors as (e.g., Tegmark [16])

$$
d(\mathbf{p})=A(\mathbf{p}) m(\mathbf{p})+n(\mathbf{p})
$$

where $d$ is the measured data, $A(\mathbf{p}), m(\mathbf{p})$, and $n(\mathbf{p})$ are, respectively, the pointing matrix, map vector with coefficients $T, Q-i U, Q+i U$, and the noise, at the pixel p. For Gaussian white noise, the optimal map $\tilde{m}$ assumes the form (Tegmark [16])

$$
\tilde{m}(\mathbf{p})=\left(\sum_{j \in \mathbf{p}} A_{j}^{T} A_{j}\right)^{-1}\left(\sum_{j \in \mathbf{p}} A_{j}^{T} d_{j}\right)
$$

where the sums run over all measurements of the pixel p, $A$ is given by

$$
A_{j}=\left(1, \frac{1}{2} e^{2 i \alpha_{j}}, \frac{1}{2} e^{-2 i \alpha_{j}}\right),
$$

and $A^{T}$ is $A$ transposed. In general the matrix elements of $A$ depend on the angle $\alpha \equiv \beta+\theta+\psi+\epsilon$ where the angles $\theta$ and $\psi$ were defined above, the angle $\epsilon$ is the sum of uncertainties in these two angles, and $\beta$ is the angle between some arbitrary axis and the $x$ axis of the focal plane. Clearly, the angular coverage uniformity of each pixel depends on the details of the scanning strategy. We address this issue in the following section.

\section{A. General Considerations}

The effects we consider in this work arise either from circular beams with unmatched main-beam full width at half maximum (FWHM; called the monopole effect) or from beams with differential ellipticities (quadrupole effect). The effect of beam ellipticity on the temperature anisotropy power spectrum was considered earlier by, e.g., Souradeep et al. [17], and the effect of beam asymmetry on the two-point correlation functions of the temperature anisotropy and polarization was considered by $\mathrm{Ng}$ [18]. Our present work, however, is multipole space based. Also, differential gain or differential pointing (dipole effect) can induce further spurious polarization signals from temperature leakage due to beam mismatch as will be discussed below. Finally, if the polarization sensitivity axes of the beams are rotated (differential rotation effect) we expect mixing between the polarization $E$ and $B$ modes, and associated leakage of power between $E$ and $B$.

We first consider a general case with $\varepsilon=0$ (i.e., no rotation error). In this case, Eq. (14) for the two-beam experiment reads

$$
\begin{aligned}
\mathbf{I}^{\prime}(\mathbf{p})= & {\left[\left(A^{T} A\right)+R_{\pi}\left(A^{T} A\right) R_{\pi}^{-1}\right]_{\mathbf{p}}^{-1} \times\left[\left(A^{T} A\right) \mathbf{I}_{1}(\mathbf{p})\right.} \\
& \left.+R_{\pi}\left(A^{T} A\right) R_{\pi}^{-1} \mathbf{I}_{2}(\mathbf{p})\right],
\end{aligned}
$$

where the vector $\mathbf{I}(\mathbf{p}) \equiv[T, Q-i U, Q+i U](\mathbf{p})$, the subscripts 1 and 2 refer to the first and second beams, respectively, and $R_{\pi}$ is the rotation matrix by $\pi$. Using Eq. (15), we obtain

$$
T^{\prime}=\left\langle T_{+}\right\rangle+\frac{1}{2}\left\langle\left(Q_{-}-i U_{-}\right) e^{2 i \alpha}\right\rangle+\frac{1}{2}\left\langle\left(Q_{-}+i U_{-}\right) e^{-2 i \alpha}\right\rangle
$$

$$
\begin{aligned}
Q^{\prime} \pm i U^{\prime}= & \frac{1}{D}\left\langle Q_{+} \pm i U_{+}\right\rangle+\frac{2}{D}\left\langle T_{-} e^{ \pm 2 i \alpha}\right\rangle \\
& +\frac{1}{D}\left\langle\left(Q_{+} \mp U_{+}\right) e^{ \pm 4 i \alpha}\right\rangle \\
& -\frac{1}{D}\left\langle Q_{+} \mp i U_{+}\right\rangle\left\langle e^{ \pm 4 i \alpha}\right\rangle \\
& -\frac{2}{D}\left\langle T_{-} e^{\mp 2 i \alpha}\right\rangle\left\langle e^{ \pm 4 i \alpha}\right\rangle \\
& -\frac{1}{D}\left\langle\left(Q_{+} \pm i U_{+}\right) e^{\mp 4 i \alpha}\right\rangle\left\langle e^{ \pm 4 i \alpha}\right\rangle,
\end{aligned}
$$

where

$$
X_{ \pm} \equiv \frac{1}{2}\left(X_{1} \pm X_{2}\right)
$$

with $X=T, Q$ or $U$ and $D \equiv 1-\left\langle e^{4 i \alpha}\right\rangle\left\langle e^{-4 i \alpha}\right\rangle$. Angular brackets stand for averaging the trigonometric functions due to the scanning strategy. To simplify the following discussion we encapsulate the properties of the scanning strategy 


$$
\begin{aligned}
f(m, n) & \equiv\left\langle e^{-i(2 m+n) \alpha}\right\rangle \\
h_{ \pm}(m, n) & \equiv \frac{1}{D}\left[f(m, n)-f(m \pm 2, n)\left\langle e^{ \pm 4 i \alpha}\right\rangle\right] .
\end{aligned}
$$

As an example of $f$ and $h_{ \pm}$, for an ideal scan strategy, the angle $\alpha$ is uniformly sampled, and as can be seen from Eq. (20), only combinations of $n$ and $m$ which satisfy $2 m+$ $n=0$ result in nonvanishing $f(m, n)$ and $h_{ \pm}(m, n)$. When the scanning strategy is nonideal, and the beam mismatch does not have the required quadrupole symmetry, the above functions $f(m, n)$ and $h_{ \pm}(m, n)$ couple to the beam mismatch to satisfy the necessary properties of spin \pm 2 fields for certain combinations of $m$ and $n$ [see Eq. (27) below]. Employing Eq. (3) we obtain the temperature anisotropy and polarization modes in Fourier space

$$
\begin{aligned}
\tilde{T}_{l}^{\prime}= & \sum_{m, n}\left[\left(B_{+}\right)_{m, n} e^{i(2 m+n) \phi_{l}} \tilde{T}_{l}\right] \star \tilde{f}_{l}(m, n)+\frac{1}{2} \sum_{m, n}\left[\left(B_{-}\right)_{m+1, n} e^{i(2 m+n) \phi_{l}}\left(\tilde{E}_{l}-i \tilde{B}_{l}\right)\right] \star \tilde{f}_{l}(m, n) \\
& +\frac{1}{2} \sum_{m, n}\left[\left(B_{-}\right)_{m-1, n} e^{i(2 m+n) \phi_{l}}\left(\tilde{E}_{l}+i \tilde{B}_{l}\right)\right] \star \tilde{f}_{l}(m, n) \\
\tilde{E}_{l}^{\prime} \pm i \tilde{B}_{l}^{\prime}= & \sum_{m, n}\left[\left(B_{+}\right)_{m, n} e^{i(2 m+n) \phi_{l}}\left(\tilde{E}_{l} \pm i \tilde{B}_{l}\right)\right] \star \tilde{h}_{ \pm l}(m, n)+\sum_{m, n}\left[\left(B_{+}\right)_{m \pm 2, n} e^{i(2 m+n) \phi_{l}}\left(\tilde{E}_{l} \mp i \tilde{B}_{l}\right)\right] \star \tilde{h_{ \pm} l}(m, n) \\
& +2 \sum_{m, n}\left[\left(B_{-}\right)_{m \pm 1, n} e^{i(2 m+n) \phi_{l}} \tilde{T}_{l}\right] \star \tilde{h_{ \pm \mathbf{l}}}(m, n),
\end{aligned}
$$

where $\star$ stands for convolution in Fourier space and $(B)_{m, n}$ are defined in Eq. (12).

The systematic power spectra are obtained by taking the angular average (in Fourier space) of the squared modulus of the expressions in Eq. (21). Because of the assumed statistical isotropy of the underlying power spectra, the celestial temperature and polarization can be taken out of the integral

$$
C_{l}^{\prime X Y} \equiv\left\langle X Y^{*}\right\rangle=\int X Y^{*} \frac{d \phi_{l}}{2 \pi}
$$

and we are left with integrations over the beam profiles in Fourier space. We use the auxiliary quantities

$$
A \equiv\left\langle T_{l}(E-i B)^{*}\right\rangle \quad C_{ \pm} \equiv\left\langle(E+i B)(E \pm i B)^{*}\right\rangle
$$

in terms of which the power spectra are

$$
\begin{gathered}
C_{l}^{\prime E}=\frac{1}{2} \operatorname{Re}\left(C_{+}+C_{-}\right) \quad C_{l}^{\prime T E}=\operatorname{Re}(A) \\
C_{l}^{\prime B}=\frac{1}{2} \operatorname{Re}\left(C_{+}-C_{-}\right) \quad C_{l}^{\prime T B}=\operatorname{Im}(A) \\
C_{l}^{\prime E B}=\frac{1}{2} \operatorname{Im}\left(C_{-}\right)
\end{gathered}
$$

and

$$
C_{l}^{\prime T}=\left\langle\left|\tilde{T}^{\prime}{ }_{l}\right|^{2}\right\rangle .
$$

The explicit forms of $A$ and $C_{ \pm}$are given in the Appendix.

Two special cases, for which these results simplify considerably, are an ideal isotropic scan (in which every pixel is being scanned many times in a random orientation so both $f(m, n)$ and $h_{ \pm}(m, n)$ identically vanish (except for the case $2 m+n=0$ ) as do their Fourier transforms $\tilde{f}(\boldsymbol{l})$ and $\tilde{h}_{ \pm}(l)$ ), and uniform coverage of the observed field (the number of hits per pixel is not large so $f$ and $h_{ \pm}$get constant, nonzero values). In this case the Fourier transforms of $f$ and $h_{ \pm}$are $\delta$-functions and there is no multipole mixing in Eq. (21). Clearly, the first case is the limit of the second when $N_{\text {hits }}$ is very large. In principle, the field of view can be few angular degrees so one may wonder if the convolution in Eq. (21) in Fourier rather than harmonic space is warranted. Indeed, all the underlying power spectra peak at high multipoles and the effects we consider here are on scales of a few percent of the beamwidth which is assumed to be $1^{\circ}$ at most and if the sky is covered relatively uniformly, the supports of the functions $\tilde{f}(\boldsymbol{l})$ and $\tilde{h_{ \pm}}(\boldsymbol{l})$ are narrow with a support on a very small range of $\Delta l$. Combining these two facts together it is evident that we can still work in the flat-sky approximation in most cases of interest.

We limit the following discussion to Gaussian beams but similar calculations can be readily done in the case of other beam shapes (at least numerically).

The corrections to the underlying power spectra are defined as follows

$$
\begin{aligned}
\Delta C_{l}^{Z} & \equiv C_{l}^{\prime Z}\left(\sigma, g_{i}, \mu_{i}, e_{i}, \rho_{i}, \varepsilon\right)-C_{l}^{\prime Z}(\sigma, 0) \\
Z & \in\{T T, T E, E E, B B, T B, E B\},
\end{aligned}
$$

which are functions of the small parameters $g, \rho, \mu, e, \varepsilon$ and the underlying power spectra, where here $Z=$

TABLE II. Definitions of the parameters associated with the dual-beam experiment.

\begin{tabular}{lcc}
\hline \hline Parameter & Beam 1 & Beam 2 \\
\hline$\sigma_{x}$ & $\sigma(1+\mu)(1+e)$ & $\sigma(1-\mu)(1+e)$ \\
\hline$\sigma_{y}$ & $\sigma(1+\mu)(1-e)$ & $\sigma(1-\mu)(1-e)$ \\
\hline$y$ & $\frac{(l \sigma)^{2}}{2}(1+\mu)^{2}\left(1+e^{2}\right)$ & $\frac{(l \sigma)^{2}}{2}(1-\mu)^{2}\left(1+e^{2}\right)$ \\
\hline$z$ & $(l \sigma)^{2}(1+\mu)^{2} e$ & $(l \sigma)^{2}(1-\mu)^{2} e$ \\
\hline \hline
\end{tabular}


TABLE III. The leading order contributions of the systematic effects to the power spectra $C_{l}^{T E}, C_{l}^{E}$, and $C_{l}^{B}$ assuming the underlying sky is not polarized (except for the rotation signal where we assume the $E$-, and $B$-mode signals are present) and general sky scanning.

\begin{tabular}{lcccc}
\hline \hline Effect & Parameter & $\Delta C_{l}^{T E}$ & $\Delta C_{l}^{E}$ & $\Delta C_{l}^{B}$ \\
\hline Gain & $g$ & 0 & $g^{2} f_{1} \star C_{l}^{T}$ & $g^{2} f_{1} \star C_{l}^{T}$ \\
\hline Monopole & $\mu$ & 0 & $4 \mu^{2}(l \sigma)^{4} f_{1} \star C_{l}^{T}$ & $4 \mu^{2}(l \sigma)^{4} f_{1} \star C_{l}^{T}$ \\
\hline Pointing & $\rho$ & $\frac{1}{2} c_{\theta} C_{l}^{T}\left[1+J_{0}(l \rho)\right] J_{2}(l \rho)-c_{\theta} J_{1}^{2}(l \rho) C_{l}^{T} \star f_{3}$ & $c_{\theta}^{2} C_{l}^{T} J_{2}^{2}(l \rho)+J_{1}^{2}(l \rho) C_{l}^{T} \star f_{2}$ & $s_{\theta}^{2} C_{l}^{T} J_{2}^{2}(l \rho)-J_{1}^{2}(l \rho) C_{l}^{T} \star f_{2}$ \\
\hline Quadrupole & $\mathrm{e}$ & $-I_{0}(z) I_{1}(z) c_{\psi} C_{l}^{T}$ & $I_{1}^{2}(z) c_{\psi}^{2} C_{l}^{T}$ & $I_{1}^{2}(z) s_{\psi}^{2} C_{l}^{T}$ \\
\hline Rotation & $\varepsilon$ & 0 & $4 \varepsilon^{2} C_{l}^{B}$ & $4 \varepsilon^{2} C_{l}^{E}$ \\
\hline \hline
\end{tabular}

$T T, T E, E E, B B, T B$, and $E B$, and as we see from Eqs. (21) and (24), the new power spectra are, in principle, combinations of all the underlying power spectra. Also, it is worth mentioning here the coupling between the various systematic effects as far as higher-order corrections are concerned. While Eqs. (21) and (12) are presented in terms of formally-exact, well-known functions, for calculations the infinite series [Eq. (12)] must be truncated, and depending on the degree of asymmetry and the physical scale in question (tantamount to the multipole number $l$ ), the number of terms in the series considered determines the accuracy of the calculation. The scalings of the leading order terms of the effects considered here are given in Tables III, $\mathrm{IV}, \mathrm{V}$, and VI assuming the underlying sky is unpolarized (except for the effect of rotation, to be discussed in the next section). In obtaining these expressions we also assumed the scanning strategy is statistically isotropic, a reasonable assumption that significantly simplifies the scaling relations we obtain. We have used the following definitions

$$
\begin{aligned}
& f_{1}=\frac{1}{2}\left|\tilde{h}_{+}(-1,0)\right|^{2} \\
& f_{2}=\frac{1}{2}\left|\tilde{h}_{+}(-1,-1)\right|^{2}+\frac{1}{2}\left|\tilde{h}_{+}(-1,1)\right|^{2} \\
& f_{3}=\frac{1}{2}\left\langle\tilde{f}(0,1) \tilde{h}_{-}^{*}(1,-1)\right\rangle,
\end{aligned}
$$

where the functions $f(m, n)$ and $h_{ \pm}(m, n)$ are defined in Eq. (20). Since the leading orders of both $J_{n}(z)$ and $I_{n}(z)$ are $\propto z^{n}$ it is clear that terminating the series at some order $n$ is equivalent to a power series expansion exact up to this order and not higher. Therefore, the calculation described here actually amounts to a power series expansion in the most general case, but simplifies considerably when either the dipole or quadrupole effects can be ignored. Tables $\mathrm{V}$ and VI show the scaling relations in the case of ideal scanning strategy corresponding to Tables III and IV, respectively. These are the irreducible signals that persist irrespective of the scanning strategy. One further remark is in order here: the coupling between the various effects is important when the parameters $l^{2} \sigma^{2} \mu, l \rho$ and $l^{2} \sigma^{2} e$ are not negligibly small compared to 1 ; in this case higherorder corrections are required and the cross terms that include the coupling between the effects cannot be ignored, but this is seldom the case. For given parameters $\mu, e$, and $\rho$, there is a "critical" value of $l$ beyond which higherorder terms become important and in this range our calculation may be particularly useful. For $\sigma \approx 1^{\circ}$ and $e, \mu$, and $\rho / \sigma \approx 0.1$ higher-order effects become important at $l \approx$ 1000 which is tantalizingly close to the scale at which the $B$-mode signal from lensing peaks (Zaldarriaga and Seljak [19], Hu [20]). However, it happens deep beyond the beam dilution scale, $l \approx 200$. Only when $\rho$ is not very small compared to $\sigma$ or $e$ and $\mu$ are not small compared to unity do these higher-order corrections contribute to the spurious polarization.

\section{B. Rotations}

We now discuss the rotation error ignored in the above treatment. Overall rotation of the two beams (cross polarization) mixes $E$ - and $B$-modes and even induces $T$ - $B$ correlation. Nonorthogonality of the beams can be described in two different ways. If only one of the two

TABLE IV. The contribution of the systematic effects to the power spectra $C_{l}^{T B}, C_{l}^{E B}$ assuming the underlying sky is not polarized (except for the rotation signal when we assume $E$-, and $B$-mode polarization are present) and general sky scanning.

\begin{tabular}{lccc}
\hline \hline Effect & Parameter & $\Delta C_{l}^{T B}$ & $\Delta C_{l}^{E B}$ \\
\hline Gain & $g$ & 0 & 0 \\
\hline Monopole & $\mu$ & 0 & 0 \\
\hline Pointing & $\rho$ & $\frac{1}{2} J_{2}(l \rho)\left[1+J_{0}(l \rho)\right] s_{\theta} C_{l}^{T}+s_{\theta} J_{1}^{2}(l \rho) C_{l}^{T} \star f_{3}$ & $s_{\theta} c_{\theta} J_{2}^{2}(l \rho) C_{l}^{T}$ \\
\hline Quadrupole & $e$ & $-I_{0}(z) I_{1}(z) s_{\psi} C_{l}^{T}$ & $I_{1}^{2}(z) s_{\psi} c_{\psi} C_{l}^{T}$ \\
\hline Rotation & $\varepsilon$ & $2 \varepsilon C_{l}^{T E}$ & $2 \varepsilon C_{l}^{E}$ \\
\hline \hline
\end{tabular}


TABLE V. The contribution of the systematic effects to the power spectra $C_{l}^{T E}, C_{l}^{E}$, and $C_{l}^{B}$ assuming the underlying sky is not polarized (except for the rotation signal where we assume the $E$-, and $B$-mode signals are present) and ideal sky scanning.

\begin{tabular}{lcccc}
\hline \hline Effect & Parameter & $\Delta C_{l}^{T E}$ & $\Delta C_{l}^{E}$ & $\Delta C_{l}^{B}$ \\
\hline Gain & $g$ & 0 & 0 & 0 \\
\hline Monopole & $\mu$ & 0 & 0 & 0 \\
\hline Pointing & $\rho$ & $\frac{1}{2}\left(1+J_{0}(l \rho)\right) J_{2}(l \rho) c_{\theta} C_{l}^{T}$ & $J_{2}^{2}(l \rho) c_{\theta}^{2} C_{l}^{T}$ & $J_{2}^{2}(l \rho) s_{\theta}^{2} C_{l}^{T}$ \\
\hline Quadrupole & $\mathrm{e}$ & $-I_{0}(z) I_{1}(z) c_{\psi} C_{l}^{T}$ & $I_{1}^{2}(z) c_{\psi}^{2} C_{l}^{T}$ & $I_{1}^{2}(z) s_{\psi}^{2} C_{l}^{T}$ \\
\hline Rotation & $\varepsilon$ & 0 & $4 \varepsilon^{2} C_{l}^{B}$ & $4 \varepsilon^{2} C_{l}^{E}$ \\
\hline \hline
\end{tabular}

detectors is misoriented by $\delta$, there is induced $E-B$ mixing. This is the approach taken by Ponthieu [10]. If the two detectors are disoriented, one by $+\delta / 2$, the other one by $-\delta / 2$, the $Q-U$ mixing induced by the first detector is compensated by that of the second detector and there is no $E-B$ mixing. In the following, we focus on the latter when we refer to nonorthogonality of the beams. We here derive the expected signal. For (overall) rotation error $\varepsilon$ and nonorthogonality measured by $\delta$ (which we split between the two beams; one is misoriented by $\delta / 2$ and the other by $-\delta / 2)$

$$
\sum_{j}\left(A^{T} d_{j}\right)(\mathbf{p})=\|M\| \mathbf{I}
$$

where $\mathbf{I} \equiv(T, Q-i U, Q+i U)$ and

$$
\|M\| \equiv\left(\begin{array}{ccc}
1 & \frac{1}{2}\left\langle e^{2 i \alpha}\right\rangle e^{2 i \varepsilon+i \delta} & \frac{1}{2}\left\langle e^{-2 i \alpha}\right\rangle e^{-2 i \varepsilon-i \delta} \\
\frac{1}{2}\left\langle e^{2 i \alpha}\right\rangle & \frac{1}{4}\left\langle e^{4 i \alpha}\right\rangle e^{2 i \varepsilon+i \delta} & \frac{1}{4} e^{-2 i \varepsilon-i \delta} \\
\frac{1}{2}\left\langle e^{-2 i \alpha}\right\rangle & \frac{1}{4} e^{2 i \varepsilon+i \delta} & \frac{1}{4}\left\langle e^{-4 i \alpha}\right\rangle e^{-2 i \varepsilon-i \delta}
\end{array}\right)
$$

and upon using Eq. (16), we obtain

$$
\left(\begin{array}{c}
T^{\prime} \\
Q^{\prime}-i U^{\prime} \\
U^{\prime}+i U^{\prime}
\end{array}\right)=\left(\begin{array}{c}
T+\frac{i}{2} \sin \delta \sum_{ \pm} F_{ \pm}(Q \mp i U) e^{ \pm 2 i \varepsilon}\left\langle e^{ \pm 2 i \alpha}\right\rangle \\
(Q-i U) \cos \delta e^{2 i \varepsilon} \\
(Q+i U) \cos \delta e^{-2 i \varepsilon}
\end{array}\right)
$$

where $F_{ \pm} \equiv \mp 1$. Employing Eqs. (3)

TABLE VI. The contribution of the systematic effects to the power spectra $C_{l}^{T B}, C_{l}^{E B}$ assuming the underlying sky is not polarized (except for the rotation signal when we assume $E$-, and $B$-mode polarization are present) and ideal sky scanning.

\begin{tabular}{lccc}
\hline \hline Effect & Parametre & $\Delta C_{l}^{T B}$ & $\Delta C_{l}^{E B}$ \\
\hline Gain & $g$ & 0 & 0 \\
\hline Monopole & $\mu$ & 0 & 0 \\
\hline Pointing & $\rho$ & $\frac{1}{2}\left[1+J_{0}(l \rho)\right] J_{2}(l \rho) s_{\theta} C_{l}^{T}$ & $s_{\theta} c_{\theta} J_{2}^{2}(l \rho) C_{l}^{T}$ \\
\hline Quadrupole & $e$ & $-I_{0}(z) I_{1}(z) s_{\psi} C_{l}^{T}$ & $I_{1}^{2}(z) s_{\psi} c_{\psi} C_{l}^{T}$ \\
\hline Rotation & $\varepsilon$ & $2 \varepsilon C_{l}^{T E}$ & $2 \varepsilon C_{l}^{E}$ \\
\hline \hline
\end{tabular}

$$
\tilde{Q}_{l} \pm i \tilde{U}_{l}=\left(E_{l} \pm i B_{l}\right) e^{\mp 2 i \phi_{l}}
$$

we obtain to leading order

$$
T_{l}^{\prime} \approx T_{l} \quad E_{l}^{\prime} \approx E_{l}-2 \varepsilon B_{l} \quad B_{l}^{\prime} \approx B_{l}+2 \varepsilon E_{l}
$$

and therefore

$$
\begin{gathered}
\Delta C_{l}^{T^{\prime}} \approx 0 \quad \Delta C_{l}^{T^{\prime} E^{\prime}} \approx 0 \quad \Delta C_{l}^{E^{\prime}} \approx 0 \\
\Delta C_{l}^{B^{\prime}} \approx 4 \varepsilon^{2} C_{l}^{E} \quad \Delta C_{l}^{T^{\prime} B^{\prime}} \approx 2 \varepsilon C_{l}^{T E} \\
\Delta C_{l}^{E^{\prime} B^{\prime}} \approx 2 \varepsilon C_{l}^{E},
\end{gathered}
$$

i.e., the leakage is a small fraction of $C_{l}^{E}$ but can still be a significant $B$-mode contaminant.

\section{Monitoring the Contamination}

A potentially useful diagnostic is the $T-B$ cross correlation. As mentioned above, this correlation function vanishes in the standard model. The spurious effects discussed in this paper cause $T$ to leak to both $E$ and $B$, and therefore the correlations $C_{l}^{T B}$ and $C_{l}^{E B}$ do not generally vanish [see Eqs. (24) and Table IV]. Furthermore, as we will see below, beam rotation can also induce $C_{l}^{T B}$ and $C_{l}^{E B}$ because rotating the telescope by $\varepsilon$ is indistinguishable from rotation of the polarization plane by $-\varepsilon$. As a result, power leaks from $T-E$ to $T-B$ and from $E$ - $E$ to $E-B$. A nonvanishing $C_{l}^{T B}$ may be attributed to an imperfect removal of the spurious polarization signals discussed in this paper. However, beam systematics are not the exclusive generating mechanisms of $C_{l}^{T B}$ and $C_{l}^{E B}$. A few physical sources of parityviolating correlations were already discussed in the literature; parity-violating terms in the Lagrangians of the electromagnetic and gravitational sectors (e.g., Lue, Wang, and Kamionkowski [21], Liu, Lee, and Ng [22], Feng et al. [23], Saito, Ichiki, and Taruya [24], Xia et al. [25], and Komatsu et al. [26]), Faraday rotation at last scattering (Kosowsky and Loeb [27]), and hypothetical primordial helical magnetic fields (Caprini, Durrer, and Kahniashvili [28]). The systematic $T$ - $B$ and $E-B$ correlations may interfere with this exotic physics and a careful analysis of these correlations in required in the presence of beam systematics (Shimon and Keating[29]). 


\section{NUMERICAL RESULTS}

The Stokes $Q$ parameters associated with the polarization due to the optical imperfections discussed in this paper are shown in Fig. 2.

We have calculated all possible six power spectra [Eqs. (24) and (33)] for two different average beam widths FWHM $56^{\prime}$ and $5^{\prime}$. Figures 3-8 depict beam rotation, differential pointing, and differential ellipticity effects on the $B$-mode power spectrum for average beamwidths of $56^{\prime}$ and $5^{\prime}$, respectively. The dot-dashed lines refer to the inflation-induced $B$-mode from primordial gravitational waves with tensor-to-scalar ratios of $T / S=10^{-1}, 10^{-2}$, $10^{-3}$, and $10^{-4}$, respectively, where we have used the definition used in CAMB for the tensor-to-scalar ratio. For the plots of the second order differential pointing effect the specified pointing error $\rho$ refers to one of the beams (we left the angle $\theta$ of this beam a free parameter), while the other beam has been assumed to have no pointing error . The quadrupole effect was calculated assuming the two beams have the same specified ellipticities, $|e|$, and here we left the angles that the polarization axes make with the major axes of the two beams, $\psi_{1}$ and $\psi_{2}$, as free parameters.

We have defined $c_{\psi} \equiv \cos \left(2 \psi_{1}\right)-\cos \left(2 \psi_{2}\right), \quad s_{\psi} \equiv$ $\sin \left(2 \psi_{1}\right)-\sin \left(2 \psi_{2}\right)$, and $c_{\theta} \equiv \cos 2 \theta, s_{\theta} \equiv \sin 2 \theta$. Note that in the case considered here, that both beams have the same ellipticity $|e|$, we obtain, as expected, that there in no spurious polarization when $\psi_{1}=\psi_{2}$. Similarly, had we assumed both beams have the same pointing $\rho$ we would have obtained no spurious polarization if $\theta_{1}=\theta_{2}$ in the case of the ideal scanning, where second order pointing is the leading order contribution (not so in the case of nonideal scanning, where the leading order is the dipole effect).

As expected, the larger the beamwidths, the larger the angular scales on which the systematics peak. The systematics are calculated for the small parameters; $g, \mu$, and $e=$ $1,2,3,4$, and $5 \%$ of the mean beam and $\rho$ and $\varepsilon$ the same fractions of the mean beam and a radian, respectively. The scaling relations of these effects are given in Tables III and IV (general scanning strategy) and V and VI (ideal scanning strategy). As explained in the last section, the higherorder corrections (beyond the leading order) are important on some scale, typically $l \approx \sigma^{-1} \mu^{-\frac{1}{2}}$ which for $\sigma \approx 1^{\circ}$ and $e$ and $\mu$ on the $1 \%$ level corresponds to $l \approx 2000$. On this scale, the $B$-mode from the CMB lensed by the large
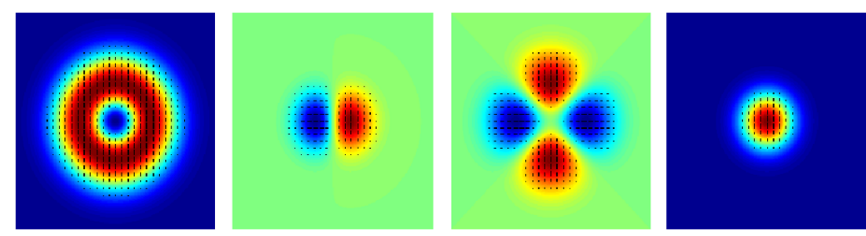

FIG. 2 (color online). An illustration of the monopole, dipole, quadrupole, and gain effects; $Q$ parameter only is depicted.

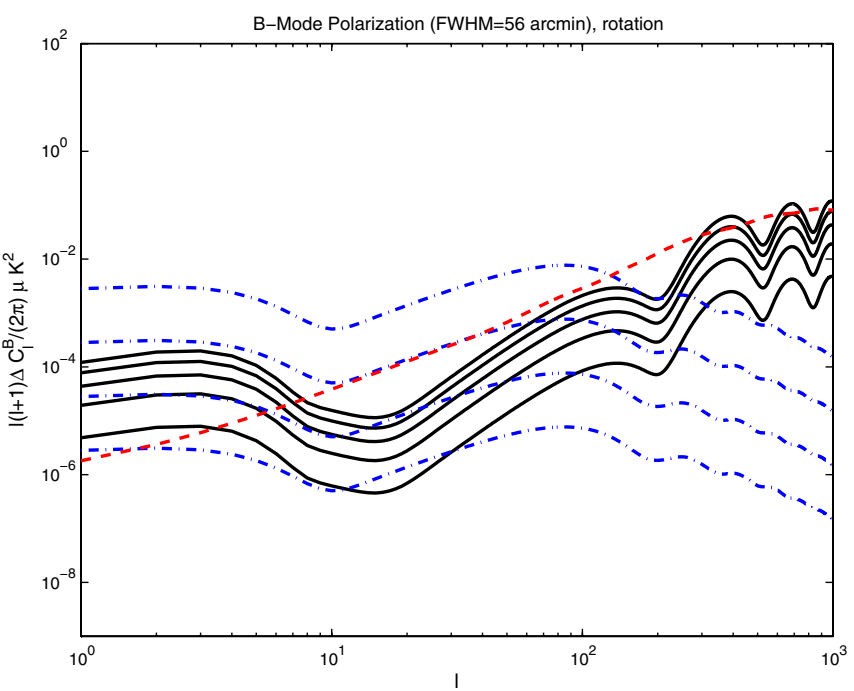

FIG. 3 (color online). The contribution of differential rotation to the $B$-mode power spectrum ( $56^{\prime}$ average beamwidth). Shown are the effects for $\varepsilon=0.01,0.02,0.03,0.04$, and 0.05 of a radian. For comparison, the dot-dashed curves refer to the contribution from primordial gravitational waves with tensor-to-scalar ratios $T / S=10^{-1}, 10^{-2}, 10^{-3}$, and $10^{-4}$. The dashed curve is the $B$-mode polarization produced by gravitational lensing by the large scale structure.

scale structure (e.g., Zaldarriaga and Seljak [19], Hu [20]) is non-negligible (the dashed lines in Figs. 3-8). However

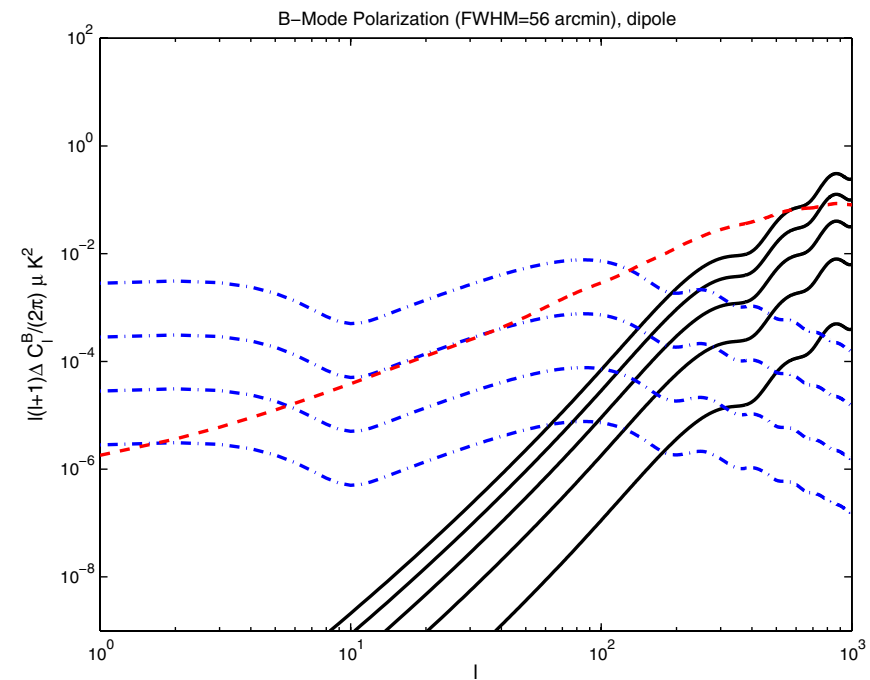

FIG. 4 (color online). The contribution of differential pointing to the $B$-mode power spectrum ( $56^{\prime}$ average beamwidth). The values shown should be multiplied by $s_{\theta}^{2}$ (Table III). In case $\theta=$ $0^{\circ}$ all the spurious polarization is in the $E$-mode. For a given $\rho$, the maximum $B$-mode is obtained at $\theta=45^{\circ}$. Shown are the effects for $\rho=0.01,0.02,0.03,0.04$, and 0.05 . For comparison, the dot-dashed curves refer to the contribution from primordial gravitational waves with tensor-to-scalar ratios $T / S=10^{-1}$, $10^{-2}, 10^{-3}$, and $10^{-4}$. The dashed curve is the $B$-mode polarization produced by gravitational lensing by the large scale structure. 


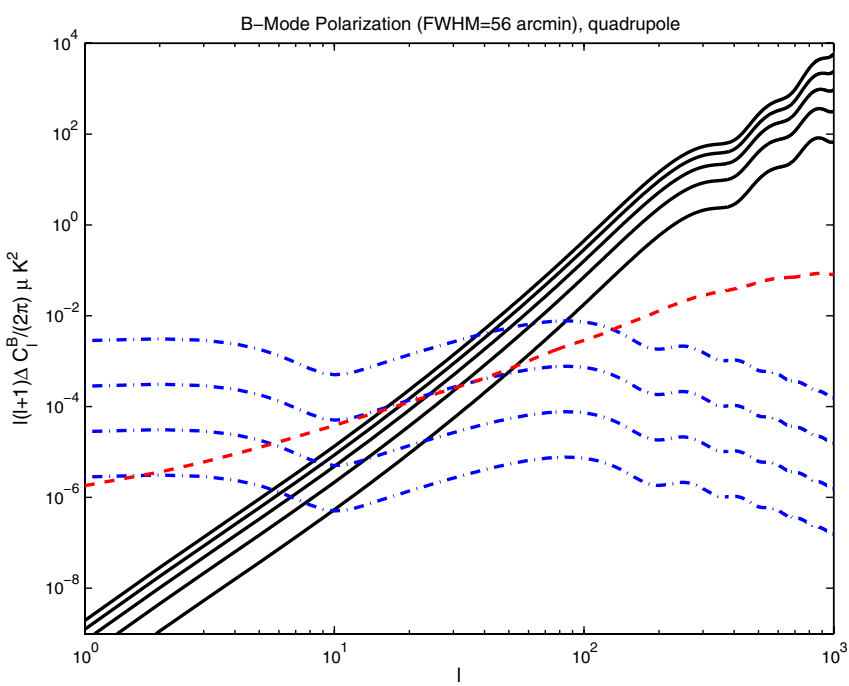

FIG. 5 (color online). The contribution of the differential beam ellipticity ("quadrupole" effect) to the $B$-mode power spectrum (56' average beamwidth). The values shown should be multiplied by $s_{\psi}^{2}$ (see Table III). Shown are the effects for $e=0.01,0.02$, $0.03,0.04$, and 0.05 . For comparison, the dot-dashed curves refer to the contribution from primordial gravitational waves with tensor-to-scalar ratios $T / S=10^{-1}, 10^{-2}, 10^{-3}$, and $10^{-4}$. The dashed curve is the $B$-mode polarization produced by gravitational lensing by the large scale structure.

a beam that size is insensitive to features on scales of $l \approx$ 2000. It is also important to mention here that the effect due to rotation (Figs. 3 and 6) closely follows the $C_{l}^{E}$ shape

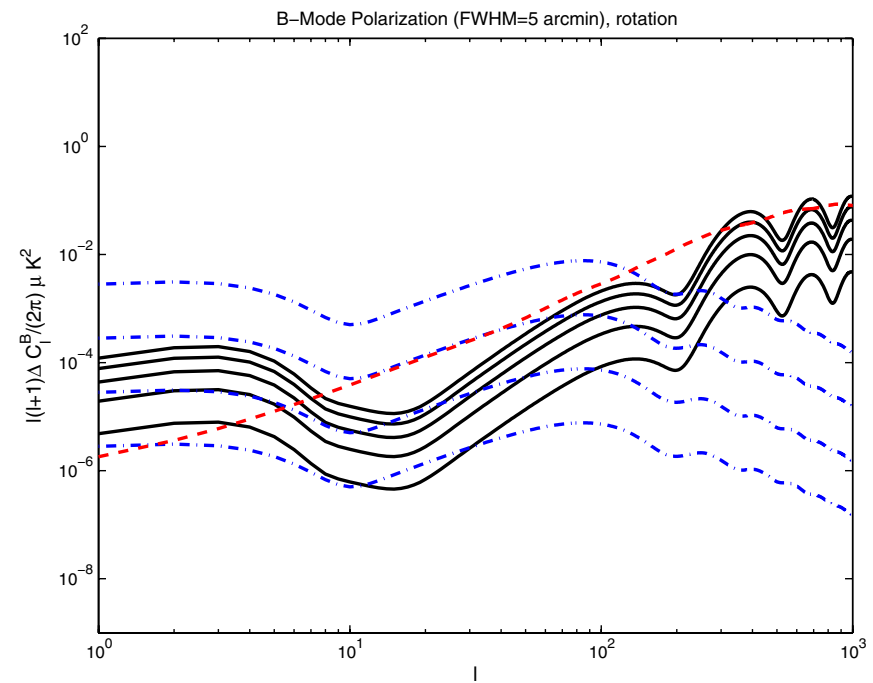

FIG. 6 (color online). The contribution of differential rotation to the $B$-mode power spectrum ( $5^{\prime}$ average beamwidth). Shown are the effects for $\varepsilon=0.01,0.02,0.03,0.04$, and 0.05 of a radian. For comparison, the dot-dashed curves refer to the contribution from primordial gravitational waves with tensor-to-scalar ratios $T / S=10^{-1}, 10^{-2}, 10^{-3}$, and $10^{-4}$. The dashed curve is the $B$-mode polarization produced by gravitational lensing by the large scale structure.

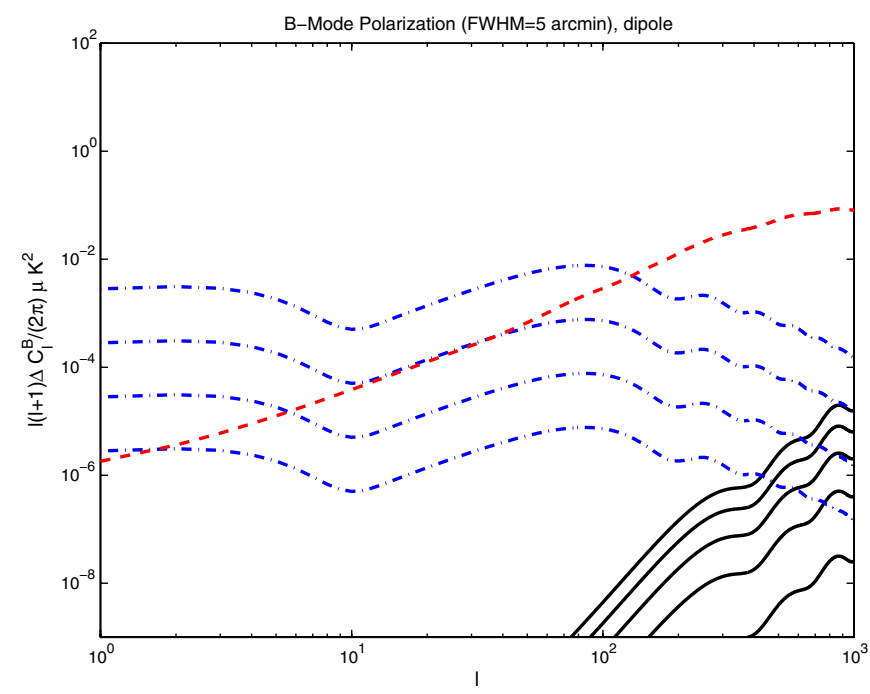

FIG. 7 (color online). The contribution of differential pointing to the $B$-mode power spectrum ( $5^{\prime}$ average beamwidth). The values shown should be multiplied by $s_{\theta}^{2}$. Shown are the effects for $\rho=0.01,0.02,0.03,0.04$, and 0.05 . For comparison, the dotdashed curves refer to the contribution from primordial gravitational waves with tensor-to-scalar ratios $T / S=10^{-1}, 10^{-2}$, $10^{-3}$, and $10^{-4}$. The dashed curve is the $B$-mode polarization produced by gravitational lensing by the large scale structure.

and merely reflects the leakage of $E$ to $B$ due to rotation [Eq. (33)]. One more remark should be made regarding the coupling of the differential pointing and quadrupole effects

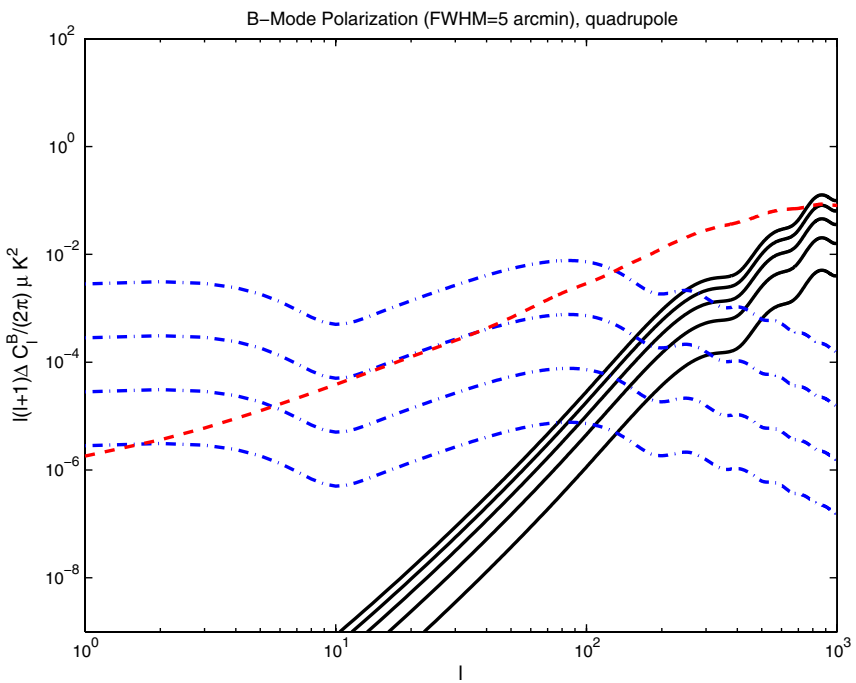

FIG. 8 (color online). The contribution of the differential beam ellipticity ("quadrupole" effect) to the $B$-mode power spectrum ( $5^{\prime}$ average beamwidth). The values shown should be multiplied by $s_{\psi}^{2}$. Shown are the effects for $e=0.01,0.02,0.03,0.04$, and 0.05 of a radian. For comparison, the dot-dashed curves refer to the contribution from primordial gravitational waves with tensor-to-scalar ratios $T / S=10^{-1}, 10^{-2}, 10^{-3}$, and $10^{-4}$. The dashed curve is the $B$-mode polarization produced by gravitational lensing by the large scale structure. 
mentioned above. As seen from Eqs. (12) the pointing error and ellipticity are coupled through products of the cylindrical and modified Bessel functions. In practice however the coupling is small since the pointing error parameter $l \rho$ is small. In this case, since the leading term of $J_{n}(x)$ is $\propto x^{n}$ it is safe to consider only the leading terms in the infinite sum in Eq. (12), this approximation considerably simplifies numerical calculations. In calculating the plots we assumed perfect scanning strategy so the monopole and gain identically vanish as expected and as can be easily verified from Eqs. (12) and (21) and the relations in the Appendix. Again, it is important to note that the power spectra for the second order pointing error (Figs. 4 and 7) and quadrupole (Figs. 5 and 8 ) should be multiplied by the functions $c_{\theta}$ and $s_{\theta}$, and $c_{\psi}$ and $s_{\psi}$, respectively, as described in Tables III, IV, V, and VI. These functions can vanish. For instance, the effect of ellipticity will not contribute to the $B$-mode power spectra if the polarization sensitive axes are parallel to one of the ellipse principal axes $(\psi=0, \pi / 2)$, there is no shear of the field in this highly symmetric case and all the spurious polarization is in the $E$-mode as can be seen from Table V. This is no longer the case with nonideal scanning strategy (Table III); in this case the first order dipole do not depend on $\theta$. Also, if $\psi_{1}=\psi_{2}$ there will be no induced polarization by differential ellipticity, not in the $E$-, nor in the $B$-mode. Similarly, for the pointing error, if $\theta=0$, all the spurious polarization will contaminate the $E$-mode (if the scanning strategy is ideal). The power spectrum associated with the underlying sky was calculated by CAMB using cosmological parameters consistent with WMAP (Spergel et al. [30]). We ignore gravitational lensing and the tensor contribution

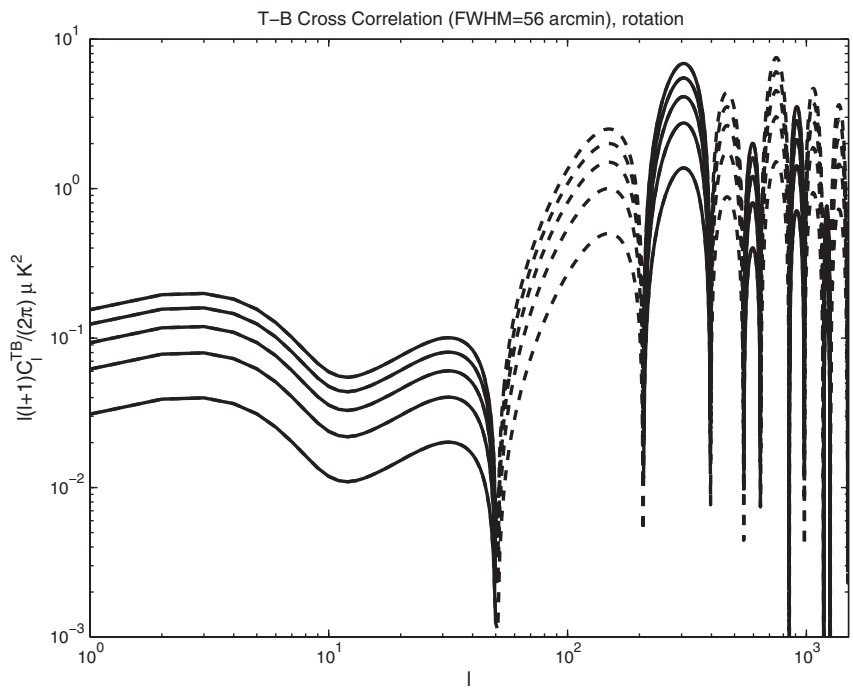

FIG. 9. The contribution of differential rotation to the $T-B$ cross correlation (56 $6^{\prime}$ average beamwidth). Shown are the signals for $\varepsilon=0.01,0.02,0.03,0.04$, and 0.05 of a radian. Dashed lines correspond to negative values of the cross correlation induced by the underlying $T-E$ cross correlation.

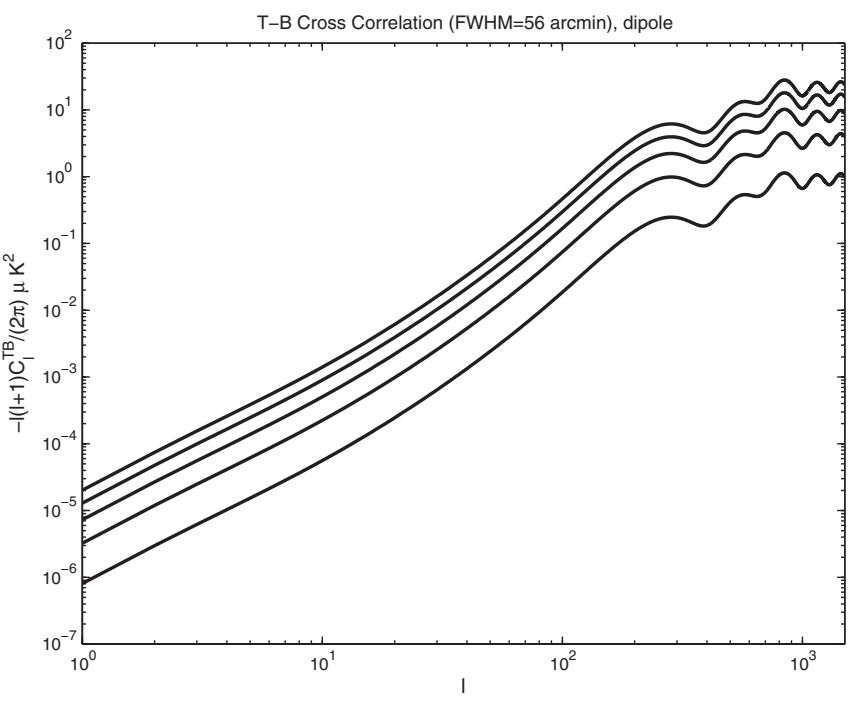

FIG. 10. The contribution of differential pointing to the $T-B$ cross correlation ( $56^{\prime}$ average beamwidth). The values shown should be multiplied by $s_{\theta}$. Shown are the effects for $\rho=0.01$, $0.02,0.03,0.04$, and 0.05 .

to the underlying sky, but we do show them in the figures for reference. We stress again that nonvanishing $C_{l}^{\prime T B}$ and $C_{l}^{\prime E B}$ may indicate an incomplete removal of the spurious polarization signals discussed here. The relevant expressions are given in Tables IV and VI. Figures 9-11 show that $C_{l}^{I T B}$ peaks at the few $\mu K$ level (all these values are for "maximum shear," i.e., $\theta=45^{\circ}$ or $\psi=45^{\circ}$ ), and so constitute only upper limits for the given parameters $\rho$ and $e$.

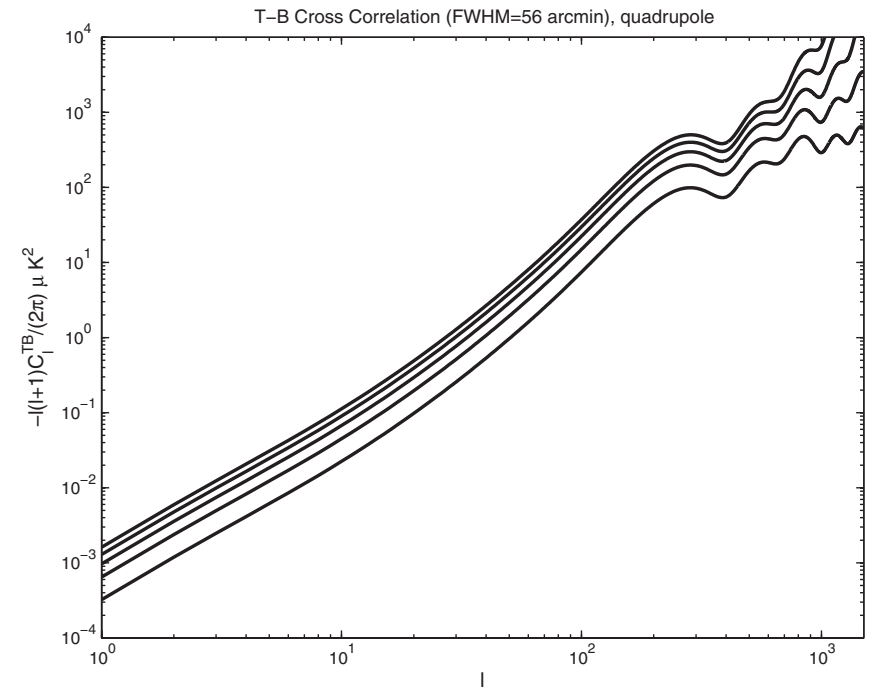

FIG. 11. The contribution of the "quadrupole" to the $T-B$ cross correlation ( $56^{\prime}$ average beamwidth). The values shown should be multiplied by $s_{\psi}$. Shown are the effects for $e=0.01$, $0.02,0.03,0.04$, and 0.05 . 
Only the contributions from (second order) differential pointing, differential ellipticity, and differential rotation for the $T B$ cross correlation are displayed since the monopole and gain contributions identically vanish for an ideal scanning strategy. One more aspect of this analysis is the higher-order corrections of the effects studied in this paper. We find that these corrections have little effect even on the smallest scales (where the gradients are large) in the ideal cases we studied. These effects become relatively large on scales much smaller than the mean beam size where beam dilution is significant. We conclude that at least for a nearly ideal scanning strategy these higher-order corrections can be safely neglected.

\section{DISCUSSION}

The systematics discussed here, more than simply affecting the peak of the $B$-mode power spectrum at $l \approx 100$ are likely to impact the polarization signal due to lensing at $l \approx$ few hundreds as shown in Figs. 3-8, at least for small beams. A full analysis of the effect of gravitational waves on CMB $B$-mode polarization is underway, and requires an assessment of the effect of gravitational lensing. The results presented in this paper show that the beam systematics are likely to further complicate this process both directly (with a residual effect on degree scales) and indirectly (by mimicking the effect of lensing). While the lensing signal can be partially extracted by invoking optimal estimators that use the non-Gaussianity of the lensed anisotropy and polarization (Hu and Okamoto [31]), the systematics discussed here cannot be removed by this method because they inherit the statistics of the underlying sky, which is Gaussian in the standard model. The lensinginduced polarization is a direct probe of structure formation processes and is sensitive to few cosmological parameters, most notably the neutrino mass. An accurate analysis should take the beam effects discussed here into account. Our calculation demonstrates that at most, these systematics are on the level of a few tens to a few hundredths of $n K$ and their significance as contaminants of the primordial $B$-mode depends on the tensor-to-scalar ratio. Comparing our results to the results of Ponthieu [10] it is evident that the spurious power spectra calculated here are less noisy because they do not include the cosmic variance.
The formalism described here directly employs the underlying power spectrum as opposed to Ponthieu [10] whose results are based on producing synthetic CMB maps and their first and second spatial derivatives with HEALPix (Górski et al. [32]). These are combined with, e.g., the first order moments of the effective beam and hits distribution in each pixel, producing simulated "observed" maps (including their systematic effects), and these maps can be studied by themselves or used to produce power spectra. Although our analytic results are given in their most general form, including the effect of scanning strategy, in practice $f_{1}, f_{2}$, and $f_{3}$ [Eq. (27)] should be numerically calculated. This is consistently done by the approach employed by Ponthieu [10]. Our analysis shows that the contributions from higher-order corrections and coupling between the various effects may be of some importance in principle, but for the cases studied here of a perfect elliptical Gaussian beam with mean beamwidth $\approx 1^{\circ}$ and ellipticity and pointing error on the $5 \%$ level with a perfect scanning strategy, we find that the higher-order corrections have a negligible effect on the angular scales of interest. Another aspect is the potential use of the nonvanishing $C_{l}^{T B}$ and $C_{l}^{E B}$ power spectra as monitors of an imperfect removal of the spurious beam effects using the fact that these two power spectra are expected to identically vanish in the standard cosmological model.

\section{ACKNOWLEDGMENTS}

We wish to acknowledge fruitful discussions with Jamie Bock and the EPIC working group. M. S. would like to thank Evan Bierman, Nathan Miller, Tom Renbarger, and John Kovac for useful discussions. B. K. gratefully acknowledges support from NSF PECASE Award No. AST-0548262 and a NASA Einstein Probe Mission Study Grant No. 785.90.00.04 "The Experimental Probe of Inflationary Cosmology (EPIC)." We acknowledge using CAMB to calculate the power spectra of the underlying sky presented in this work.

\section{APPENDIX: AUXILIARY CORRELATION FUNCTIONS}

We list here the auxiliary correlation functions $A$ and $C_{ \pm}$

$$
\begin{aligned}
& C_{ \pm} \equiv\left\langle(E+i B)(E \pm i B)^{*}\right\rangle \\
& =\sum_{m n m^{\prime} n^{\prime}}\left\langle\tilde { h } _ { + } ( m , n ) \tilde { h } _ { \pm } ^ { * } ( m ^ { \prime } , n ^ { \prime } ) e ^ { i [ 2 ( m - m ^ { \prime } ) + ( n - n ^ { \prime } ) ] \phi _ { l } \rangle } \star \left[ 4 C_{l}^{T}\left(B_{-}\right)_{m+1, n}\left(B_{-}\right)_{m^{\prime} \pm 1, n^{\prime}}^{*}+2 C_{l}^{T E}\left[( B _ { - } ) _ { m + 1 , n } \left(\left(B_{+}\right)_{m^{\prime} n^{\prime}}\right.\right.\right.\right. \\
& \left.\left.+\left(B_{+}\right)_{m^{\prime} \pm 2, n^{\prime}}\right)^{*}+\left(B_{-}\right)_{m^{\prime} \pm 1, n^{\prime}}^{*}\left(\left(B_{+}\right)_{m, n}+\left(B_{+}\right)_{m+2, n}\right)\right]+C_{l}^{E}\left[\left(B_{+}\right)_{m^{\prime} n^{\prime}}^{*}+\left(B_{+}\right)_{m^{\prime} \pm 2, n^{\prime}}^{*}\right] \times\left[\left(B_{+}\right)_{m, n}+\left(B_{+}\right)_{m+2, n}\right] \\
& \pm C_{l}^{B}\left(\left(B_{+}\right)_{m, n}-\left(B_{+}\right)_{m+2, n}\right) \times\left(\left(B_{+}\right)_{m^{\prime}, n^{\prime}}^{*}-\left(B_{+}\right)_{m^{\prime} \pm 2, n^{\prime}}^{*} \pm 2 i C_{l}^{T B}\left[\left(B_{-}\right)_{m+1, n}\left(\left(B_{+}\right)_{m^{\prime} \pm 2, n^{\prime}}-\left(B_{+}\right)_{m^{\prime}, n^{\prime}}\right)^{*}\right.\right. \\
& \left.\left. \pm\left(B_{-}\right)_{m^{\prime} \pm 1, n^{\prime}}^{*}\left(B_{+}\right)_{m, n}-\left(B_{+}\right)_{m+2, n}\right)\right] \pm i C_{l}^{E B}\left[\left(\left(B_{+}\right)_{m, n}+\left(B_{+}\right)_{m+2, n}\right) \times\left(\left(B_{+}\right)_{m^{\prime} \pm 2, n^{\prime}}^{*}-\left(B_{+}\right)_{m^{\prime}, n^{\prime}}^{*}\right)\right. \\
& \left. \pm\left(\left(B_{+}\right)_{m, n}-\left(B_{+}\right)_{m+2, n}\right) \times\left(\left(B_{+}\right)_{m^{\prime} \pm 2, n^{\prime}}^{*}+\left(B_{+}\right)_{m^{\prime}, n^{\prime}}^{*}\right)\right] \text {. }
\end{aligned}
$$




$$
\begin{aligned}
& A \equiv\left\langle T(E-i B)^{*}\right\rangle \\
& =\sum_{m n m^{\prime} n^{\prime}}\left\langle\tilde{f}(m, n) \tilde{h}_{-}^{*}\left(m^{\prime}, n^{\prime}\right) e^{i\left[2\left(m-m^{\prime}\right)+\left(n-n^{\prime}\right)\right] \phi_{l}}\right\rangle \star\left[2 C_{l}^{T}\left(B_{+}\right)_{m n}\left(B_{-}\right)_{m^{\prime}-1, n^{\prime}}^{*}+C_{l}^{T E}\left[\left(B_{+}\right)_{m n}\left(\left(B_{+}\right)_{m^{\prime} n^{\prime}}+\left(B_{+}\right)_{m^{\prime}-2, n^{\prime}}\right)^{*}\right.\right. \\
& \left.+\left(B_{-}\right)_{m^{\prime}-1, n^{\prime}}^{*}\left(\left(B_{-}\right)_{m+1, n}+\left(B_{-}\right)_{m-1, n}\right)\right]+\frac{1}{2} C_{l}^{E}\left[\left(B_{+}\right)_{m^{\prime} n^{\prime}}^{*}+\left(B_{+}\right)_{m^{\prime}-2, n^{\prime}}^{*}\right] \times\left[\left(B_{-}\right)_{m+1, n}+\left(B_{-}\right)_{m-1, n}\right] \\
& +\frac{1}{2} C_{l}^{B}\left[\left(B_{+}\right)_{m^{\prime}, n^{\prime}}^{*}-\left(B_{+}\right)_{m^{\prime}-2, n^{\prime}}^{*}\right] \times\left[\left(B_{-}\right)_{m+1, n}-\left(B_{-}\right)_{m-1, n}\right]+i C_{l}^{T B}\left[\left(B_{+}\right)_{m n}\left(\left(B_{+}\right)_{m^{\prime}, n^{\prime}}-\left(B_{+}\right)_{m^{\prime}-2, n^{\prime}}\right)^{*}\right. \\
& \left.+\left(B_{-}\right)_{m^{\prime}-1, n^{\prime}}^{*}\left(\left(B_{-}\right)_{m-1, n}-\left(B_{-}\right)_{m+1, n}\right)\right]+\frac{i}{2} C_{l}^{E B}\left[\left(\left(B_{-}\right)_{m+1, n}+\left(B_{-}\right)_{m-1, n}\right) \times\left(\left(B_{+}\right)_{m^{\prime} n^{\prime}}^{*}-\left(B_{+}\right)_{m^{\prime}-2, n^{\prime}}^{*}\right)\right. \\
& \left.\left.-\left(\left(B_{-}\right)_{m+1, n}-\left(B_{-}\right)_{m-1, n}\right) \times\left(\left(B_{+}\right)_{m^{\prime}, n^{\prime}}^{*}+\left(B_{+}\right)_{m^{\prime}-2, n^{\prime}}^{*}\right)\right]\right] \text {, }
\end{aligned}
$$

where $\star$ denotes $2 \mathrm{D}$ convolution.

[1] U. Seljak and M. Zaldarriaga, Phys. Rev. Lett. 78, 2054 (1997).

[2] M. Kamionkowski, A. Kosowsky, and A. Stebbins, Phys. Rev. Lett. 78, 2058 (1997).

[3] M. Zaldarriaga, Phys. Rev. D 55, 1822 (1997).

[4] X. Fan, C. L. Carilli, and B. Keating, Annu. Rev. Astron. Astrophys. 44, 415 (2006).

[5] A. Amblard, A. Cooray, and M. Kaplinghat, Phys. Rev. D 75, 083508 (2007).

[6] A. Lewis, A. Challinor, and N. Turok, Phys. Rev. D 65, 023505 (2002).

[7] A. de Oliveira-Costa and M. Tegmark, Phys. Rev. D 74, 023005 (2006).

[8] M. L. Brown, P. G. Castro, and A. N. Taylor, Mon. Not. R. Astron. Soc. 360, 1262 (2005).

[9] W. Hu, M. M. Hedman, and M. Zaldarriaga, Phys. Rev. D 67, 043004 (2003).

[10] N. Ponthieu (private communication).

[11] C. Rosset et al., arXiv: astro-ph/0410544.

[12] D. O’Dea, A. Challinor, and B. R. Johnson, Mon. Not. R. Astron. Soc. 376, 1767 (2007).

[13] S. Masi et al., Astron. Astrophys. 458, 687 (2006).

[14] A. Lewis, A. Challinor, and A. Lasenby, Astrophys. J. 538, 473 (2000).

[15] M. Zaldarriaga and U. Seljak, Phys. Rev. D 55, 1830 (1997).

[16] M. Tegmark, Phys. Rev. D 56, 4514 (1997).
[17] T. Souradeep, S. Mitra, A. Sengupta, S. Ray, and R. Saha, New Astron. Rev. 50, 1030 (2006).

[18] K.-W. Ng, Phys. Rev. D 71, 083009 (2005).

[19] M. Zaldarriaga and U. Seljak, Phys. Rev. D 58, 023003 (1998).

[20] W. Hu, Phys. Rev. D 62, 043007 (2000).

[21] A. Lue, L. Wang, and M. Kamionkowski, Phys. Rev. Lett. 83, 1506 (1999).

[22] G.-C. Liu, S. Lee, and K.-W. Ng, Phys. Rev. Lett. 97, 161303 (2006).

[23] B. Feng, M. Li, J.-Q. Xia, X. Chen, and X. Zhang, Phys. Rev. Lett. 96, 221302 (2006).

[24] S. Saito, K. Ichiki, and A. Taruya, J. Cosmol. Astropart. Phys. 09 (2007) 2.

[25] J.-Q. Xia, H. Li, X. Wang, and X. Zhang, arXiv:0710.3325.

[26] E. Komatsu et al., arXiv:0803.0547.

[27] A. Kosowsky and A. Loeb, Astrophys. J. 469, 1 (1996).

[28] C. Caprini, R. Durrer, and T. Kahniashvili, Phys. Rev. D 69, 063006 (2004).

[29] M. Shimon and B. Keating (work in progress).

[30] D. N. Spergel et al., Astrophys. J. 170, 377 (2007).

[31] W. Hu and T. Okamoto, Astrophys. J. 574, 566 (2002).

[32] K. M. Górski, E. Hivon, A. J. Banday, B. D. Wandelt, F. K. Hansen, M. Reinecke, and M. Bartelmann, Astrophys. J. 622, 759 (2005). 